\title{
Premorbid intelligence of inpatients with different psychiatric diagnoses does not differ
}

\author{
Paolo Stratta' \\ Ilaria Riccardi ${ }^{2}$ \\ Annarita Tomassini ${ }^{2}$ \\ Maria Marronaro² \\ Roberta Pacifico ${ }^{2}$ \\ Alessandro Rossi $\mathrm{i}^{2,3}$ \\ 'Department of Mental Health, \\ A.U.S.L. 4 L'Aquila, Italy; ${ }^{2}$ Department \\ of Experimental Medicine, University \\ of L'Aquila, Italy; ${ }^{3}$ Clinical Psychology \\ Unit at Villa Serena, c/o 'Casa di \\ Cura Villa Serena', Viale L. Petruzzi, \\ 19, Città S.Angelo, Pescara, Italy
}

\begin{abstract}
The diagnostic specificity of poor premorbid intelligence is controversial. We explored premorbid intelligence level in psychiatric patients with personality disorders, depressive disorders, bipolar disorders and schizophrenic disorders. 273 consecutively admitted patients and 81 controls were included in the study and tested with the 'Test di Intelligenza Breve', an Italian adaptation of the National Adult Reading Test. Significant differences between the clinical samples and the control subjects were found but not among the 4 clinical groups. The observation of premorbid IQ deficits in subjects with diagnoses other than schizophrenia suggests a common vulnerability diathesis, which is most likely to have a neurodevelopmental basis.
\end{abstract}

Keywords: premorbid intelligence, psychiatric disorders, specificity

\section{Introduction}

Although poor premorbid intelligence among subjects with schizophrenia is a consistent finding (Woodberry et al 2008), there are still some controversies about its diagnostic specificity. There are few data on premorbid cognitive functioning in subjects with diagnoses other than schizophrenia, reporting conflicting results. Cannon et al (1997) reported subjects with schizophrenia more impaired on premorbid IQ scores than those suffering from affective disorders. In a prospective study, van Os et al (1997) demonstrated that subjects with chronic affective illnesses were more cognitively impaired during childhood and adolescence than controls. Others studies have shown no or few differences between subjects with schizophrenia and non-psychotic psychiatric disorders (Isohanni et al 1998).

More recently Mortensen et al (2005) reported that schizophrenia and related disorders, other psychotic disorders, adjustment, personality, alcohol and substance use related disorders but not mood disorders as well as neuroses and related disorders were significantly associated with low IQ scores. On the other hand, results of Tiihonen et al (2005) indicate that premorbid intellectual ability, in terms of visuospatial reasoning, is impaired in bipolar disorder and schizophrenia and, to a smaller extent, in other psychoses suggesting a possible shared aetiology.

Several studies finally report that the association between cognitive deficits and functional impairment may exist across most diagnostic entities (Zammit et al 2004; Weiser et al 2004).

There is therefore a rationale for further studies examining whether low IQ test scores are specifically associated with schizophrenia or are a marker of increased vulnerability to the development of a broader range of psychiatric disorders.

In the present study we aimed to investigate the premorbid intelligence level across diagnoses in psychiatric patients during an index admission. Lacking IQ evaluations before the illness onset, we used the 'Test di Intelligenza Breve' (TIB), Italian adaptation of the National Adult Reading Test (NART) (Sartori et al 1997; Nelson 1982). This instrument is based on reading task, considered a valid 
and clinically useful method to investigate premorbid intellectual abilities (Crawford et al 1990; Griffin et al 2002; Stratta et al 2007).

\section{Method}

\section{Clinical sample}

Consecutively admitted patients to Villa Serena Medical Centre, Psychiatry Unit, Città S. Angelo (Italy) were included if they fulfilled the following criteria: 1) informed consent to participate in the study; 2) less than 55 years of age; and 3) no history of organic brain disease, 4) mood, personality and schizophrenic disorders. Subjects were diagnosed according to DSM-IV criteria and all available clinical and historical information collected during the hospital stay, by two senior psychiatrists (AR and PS), who personally interviewed the patients.

The local Ethics Committee approved the study procedures. All subjects entering the protocol provided informed consent after receiving a complete description of the study.

A total of 273 subjects were enrolled in the study: 79 (28.94\%) women and 194 men, with a mean age of 43.31 years (SD 13.97). Educational level was 9.92 (SD 3.84). DSM-IV diagnoses were personality disorder $(\mathrm{N}=56)$, depressive disorder $(\mathrm{N}=51)$, bipolar disorder $(\mathrm{N}=64)$, schizophrenic disorder $(\mathrm{N}=102)$.

Because of high cluster A, B and C comorbidity, we decided to group personality disorders together. No other diagnoses were a priori considered for IQ evaluation in so far as only marginally represented among inpatient sample. The referral centre does not admit dual diagnoses.

\section{Control subjects}

Control subjects were recruited from the local community [81 subjects, 50 women and 31 men; mean age 40.42 (SD 9.26), educational level 12.51 (SD 3.32)].

Because the patient group may have had a decline in cognitive function, an effort was made to match patient and control groups as closely as possible on age and socioeconomic status. Thus, the control data provide an estimate of the cognitive function level expected in individuals with the same socioeconomic background of the patient group.

\section{Procedure}

Subjects were tested using the 'Test di Intelligenza Breve' (TIB) (Sartori et al 1997) just before discharge, in a remission phase. The TIB consists of 54 words (34 effective test-words with irregular accent and 20 control-words with high frequency of use), that subjects have to read and pronounce. The total number of mistakes of reading defines the TIB error score. The estimated IQ scores (ie, performance, verbal and total) are calculated through the regression of equations taking into account sex, age and educational level: estimated performance $\mathrm{IQ}=(0.194 \times$ age $)+(-1.154 \times$ TIB errors $)+$ 107.364; estimated verbal $\mathrm{IQ}=(4.198 \times \operatorname{sex} \mathrm{F}=0 \mathrm{M}=1)+$ $(0.938 \times$ educational level $)+(-0.893 \times$ TIB errors $)+99.536$; estimated total IQ $=(0.659 \times$ educational level $)+$ $(-0.947 \times$ TIB errors $)+107.58$.

The Clinical Global Impression-Severity (CGI-S) (Guy 1976) and the Global Assessment of Functioning (GAF) Scale (DSM IV, Axis V, APA 1995) for the assessment of the psychological, social, and occupational functioning were obtained for patient groups.

\section{Results}

Sex distribution was different, but no between sex comparisons reached significance. No differences in age and educational level were seen among the four clinical groups. No differences for age between clinical sample and healthy subjects were seen. However controls were more educated (Table 1).

The data of the clinical as well as the control samples were appreciably normally distributed (Kolmogorov-Smirnov tests from 0.65 to 1.18 , all not significant). One way ANOVAs on estimated IQs showed significant differences between the clinical samples and the control subjects (Scheffé tests all significant at 0.0005 level), without any difference between them (Table 1).

Correlations among educational level and estimated IQs have been performed: educational level vs Total IQ $r=0.83$, vs Performance IQ $r=0.65$; vs Verbal IQ $r=0.84$, all $p<0.0005$. Although educational level was included in the estimated IQs calculation, we tentatively considered this variable as covariate. No differences were seen among the clinical samples and IQs remained significantly different between patients and controls (Table 1).

CGI and GAF evaluations were performed to get more information on the clinical variability: subjects with schizophrenia only differed from the other samples with more severe disorder and impaired functioning (Table 1).

\section{Discussion}

The lack of premorbid IQ level specificity among different diagnoses, such as schizophrenia, affective and personality disorders confirms results of recent studies (Zammit et al 2004; Weiser et al 2004; Mortensen et al 2005). 
Table I Socio-demographic, clinical characteristics and estimated IQs in the clinical samples and in control subjects [mean (SD)]

\begin{tabular}{|c|c|c|c|c|c|c|c|c|}
\hline & $\mathbf{N}$ & Age & $\begin{array}{l}\text { Educational } \\
\text { level }\end{array}$ & CGI-S & GAF & Total IQ & Verbal IQ & $\begin{array}{l}\text { Perfor-mance } \\
\text { IQ }\end{array}$ \\
\hline Personality disorders & 56 & $40.81(10.38)$ & $8.91(3.5 I)$ & $3.9(1.2)$ & $66.0(12.0)$ & $100.14(9.64)$ & $98.79(10.36)$ & $99.06(9.39)$ \\
\hline Depressive disorder & 51 & 46.77 (I4.42) & $8.33(4.06)$ & $3.5(0.9)$ & $70.0(9.1)$ & $100.11(11.61)$ & $97.68(12.86)$ & $100.64(9.95)$ \\
\hline Bipolar disorder & 64 & $43.88(12.52)$ & $9.60(3.93)$ & $3.7(1.0)$ & $65.9(10.5)$ & $101.76(10.61)$ & $99.58($ (II.5I) & $101.07(10.48)$ \\
\hline Schizophrenic disorder & 102 & 42.60 (15.99) & 9.79 (3.77) & $4.5(1.2)$ & $55.8(12.5)$ & $102.12(10.32)$ & $100.74(10.80)$ & 101.11 (10.17) \\
\hline Control subjects & 81 & $40.42(9.26)$ & $|2.5|(3.32)$ & & & III.20 (5.40) & $109.58(6.78)$ & $109.44(4.56)$ \\
\hline One way ANOVA & & $\begin{array}{l}F=2.30 \\
N S\end{array}$ & $\begin{array}{l}F=13.25 \\
P<0.0005 \S\end{array}$ & $\begin{array}{l}\mathrm{F}=12.18 \\
\mathrm{P}<0.0005^{*}\end{array}$ & $\begin{array}{l}\mathrm{F}=22.75 \\
\mathrm{P}<0.0005^{*}\end{array}$ & $\begin{array}{l}F=17.45 \\
P<0.0005 \S\end{array}$ & $\begin{array}{l}F=15.41 \\
P<0.0005 \S\end{array}$ & $\begin{array}{l}F=15.41 \\
P<0.0005 \S\end{array}$ \\
\hline One way $\mathrm{ANCOVA}^{+}$ & & & & & & $\begin{array}{l}F=5.03 \\
P<0.001\end{array}$ & $\begin{array}{l}F=3.09 \\
P<0.025\end{array}$ & $\begin{array}{l}F=5.26 \\
P<0.005\end{array}$ \\
\hline $\begin{array}{l}\text { Regression analysis for } \\
\text { covariates }\end{array}$ & & & & & & & & \\
\hline Educational level & & & & & & $\begin{array}{l}t=25.72 \\
p<0.0005\end{array}$ & $\begin{array}{l}t=26.78 \\
P<0.0005\end{array}$ & $\begin{array}{l}t=14.27 \\
P<0.0005\end{array}$ \\
\hline
\end{tabular}

Scheffé tests.

*Subjects with schizophrenia vs the other clinical groups differ at a 0.05 level (d.f. 3.272).

${ }^{\S}$ Clinical samples vs control subjects: all significant at a 0.0005 level (d.f. 4,353).

+Educational level as covariate (d.f. 4,353).

These data suggest a vulnerability diathesis likely having a neurodevelopmental origin such as sub optimal brain development. This vulnerability diathesis can lead to a mental disorder due to attentional difficulties, distorted perceptions and cognitive interpretation of stimuli which is precipitated possibly by distressing events leading to unusual thought processes, decreased empathic ability and social functioning (Mueser and McGurk 2004).

Even though we are aware that other factors could have influenced IQ performance (ie, medication, time of testing), we attempted to consider the role of general factors affecting clinical outcome using CGI-S and GAF. Subjects with schizophrenia did have a more severe disorder but similar to other groups premorbid IQ. Different genetic and environmental factors interacting with premorbid IQ can orient towards different phenotypical outcomes (ie, diagnoses, severity of illness, global functioning) (Gottesman and Gould 2003).

However, premorbid IQ is associated with poorer outcome and higher mortality (Munro et al 2002; Batty et al 2007) and it has also been shown to have a pathoplastic effect on symptom presentation in functional psychoses (Stratta et al 2007), ie, influencing the symptom expression during an index episode. The premorbid IQ, among other factors, could therefore affect this 'intermediate outcome' and, having an effect on symptomatology, obscure diagnostic boundary.

The lack of inclusion of patients with other diagnoses limits the conclusions of this study to the sample described. The role of premorbid IQ in outpatients with other diagnoses deserves further investigation.
Although not surprising and not the primary aim of the study, the observation of a difference between controls and patients deserves some comments.

Several reasons could lead to overestimate a between group difference. The choice of a not adequate control sample could be the first. Although we selected a control group of similar age, socioeconomic status and environment, the educational level was different.

The relevance of educational level to neurocognitive measures has been widely discussed in the literature (Stratta et al 2001). It is conceivable that the illness onset could limit further achievement, thus contaminating the measure of educational level. Equating patients and controls on a variable (ie, educational level) that is descriptive of the disease outcome process may lead to a 'matching fallacy' (Meheel 1970), with the risk of biased comparisons, contrasting overachieving patients with underachieving controls (Rossi et al 1991).

However, when educational level was controlled by covariance, results were similar and, above all, no differences among patients emerged. Moreover the data we reported are in line with those of premorbid IQ estimated in similar control samples obtained by different tools: TIB (Sartori et al 1997), NART (eg, Kerr et al 2003), WAIS-R (eg, O'Leary et al 2000). It could be possible that recruiting admitted patients produces a bias with less severe patients and putatively less impaired IQ excluded: if subjects with low IQ are more likely than subjects with high IQ to be admitted to hospital, an overestimation of an association between low IQ and 
disorder could occur. However Zammit et al (2004) did not find association between IQ and illness severity but we reported that premorbid IQ could influence symptoms presentation (Stratta et al 2007).

On the other hand the differences between patients and controls could be underestimated: tests of reading ability (eg, NART) provide an accurate estimate of premorbid IQ for individuals in the average range of intelligence, while it appears of relatively lower utility for individuals at the lower end of IQ range due to the overestimation of their performance (Griffin et al 2002).

Preventive intervention on more susceptible people could be an important aim, and evaluation of cognitive abilities in adolescence or early adulthood a useful tool to identify at risk individuals (Verdoux and Liraud 2000).

\section{Disclosures}

The authors have no conflicts of interest to disclose.

\section{References}

American Psychiatric Association. 1995. Diagnostic and statistical manual of mental disorders, fourth edition: DSM-IV axis V. American Psychiatric Association.

Batty GD, Deary IJ, Gottdfredson LS. 2007. Premorbid (early life) IQ and Later Mortality Risk: Systematic Review. Ann Epidemiol, 17:278-88.

Cannon M, Jones P, Gilvarry C, et al. 1997. Premorbid social functioning in schizophrenia and bipolar disorder: similarities and differences. Am J Psychiatry, 154:1544-50.

Crowford JR, Hart S, Nelson HE. 1990. Improved detection of cognitive impairment with the NART: An investigation employing hierarchical discriminant function analysis. Br J Clin Psychol, 29:239-41.

Gottesman II, Gould TD. 2003. The Endophenotype Concept in Psychiatry: Etymology and Strategic Intentions. Am J Psychiatry, 160:636-45.

Griffin S, Mindt MR, Rankin EJ, et al. 2002. Estimating premorbid intelligence comparison of traditional and contemporary methods across the intelligence continuum. Arch Clin Neuropsychol, 17:497-07.

Guy W. 1976. Clinical global impression. In:',, (ed). ECDEU assessment manual for psychopharmacology. US Dept. of Health, Education, and Welfare publication ADDM 76-338. Rockville, MD: National Institute of Mental Health. pp. 218-22.
Isohanni I, Jarvelin MR, Nieminen PM, et al. 1998. School performance as a predictor of psychiatric hospitalization in adult life. A 28-year follow-up in the Nothern Finland 1966 Bith Cohort. Psychol Med, 28:967-74.

Kerr N, Dunbar RIM, Bentall RP. 2003. Theory of mind deficits in bipolar affective disorders. J Affect Disord, 73:253-59.

Meehl PE. 1970. Nuisance variables and the ex post facto design. In: Radner M, Winokur S (eds), Minnesota Studies in the Philosophy of Science. University of Minnesota Press, Minneapolis, pp. 373-402.

Mortensen EL, Sorensen HJ, Jensen HH, et al. 2005. IQ and mental disorder in young men. Br J Psychiatry, 187:407-15.

Mueser KT, McGurk SR. 2004. Schizophrenia. Lancet, 363:2063-72.

Munro JC, Russell AJ, Murray RM, et al. 2002. IQ in childhood psychiatric attendees predicts outcome of later schizophrenia at 21 year follow-up. Acta Psychiatr Scand, 106(2):139-42.

Nelson HE. 1982. The National Adult Reading Test (NART): test manual. In: Nelson HE (ed). NFER-Nelson Publishing. p 1-13.

O'Leary DS, Flaum M, Kesler ML, et al. 2000. Cognitive correlates of the negative, disorganized, and psychotic symptom dimensions of schizophrenia. J Neuropsychiatry Clin Neurosci, 12:4-15.

Rossi A, Stratta P, de Cataldo S, et al. 1991. Lateral ventricular size, educational level and patient subtypes in schizophrenia. BrJ Psychiatry 159: 443-4.

Sartori G, Colombo L, Vallar G, et al. 1997. T.I.B.: Test di Intelligenza Breve per la valutazione del quoziente intellettivo attuale e pre-morboso. La Professione di Psicologo, 1:II-XXIV (inserto).

Stratta P, Prosperini P, Daneluzzo E et al. 2001. Educational level and age influence spatial working memory and Wisconsin Card Sorting Test performance differently: a controller study in schizophrenic patients. Psychiatry Res, 102: 39-48.

Stratta P, Rinaldi O, Daneluzzo E, et al. 2007. Does premorbid IQ have a pathoplastic effect on symptom presentation in functional psychoses? Encephale, 33:733-37.

Tiihonen J, Haukka J, Henriksson M, et al. 2005. Premorbid intellectual functioning in bipolar disorder and schizophrenia: results from a cohort study of male conscripts. Am J Psychiatry, 162:1904-10.

Van Os J, Jones PB, Lewis G, et al. 1997. Developmental precursors of affective illness in a general population birth cohort. Arch Gen Psychiatry, 54:625-31.

Verdoux H, Liraud F. 2000. Neuropsychological function in subjects with psychotic and affective disorders. Relationship to diagnostic category and duration of illness. Eur Psychiatry, 15:236-43.

Weiser M, Reichenberg A, Rabinowitz J, et al. 2004. Cognitive performance of male adolescents is lower than controls across psychiatric disorders: a population- based study. Acta Psychiatr Scand, 110:471-75.

Woodberry KA, Giuliano Aj, Seidman LJ. 2008. Premorbid IQ in Schizophrenia: A Meta-Analytic Review. Am J Psychiatry, 165:579-87.

Zammit S, Allebeck P, David AS, et al. 2004. A longitudinal study of premorbid IQ scores and risk of developing schizophrenia, bipolar disorder, severe depression, and other nonaffective psychoses. Arch Gen Psychiatry, 61:354-60. 